\title{
Pathophysiology of Graves' Ophthalmopathy: A Literature Review
}

\author{
Mariela Carolina Santos Carballo1, Bianca Paiva Cunha de Sá1, \\ Denise Rosso Tenório Wanderley Rocha ${ }^{1}$, Alberto Krayyem Arbex ${ }^{1,2}$ \\ ${ }^{1}$ Division of Endocrinology, IPEMED Medical School, São Paulo, Brazil \\ ${ }^{2}$ Diabetology Department, Malteser Krankenhaus St. Franziskus-Hospital, Flensburg, Germany \\ Email: mariela.carballo@gmail.com
}

How to cite this paper: Carballo, M.C.S. de Sá, B.P.C., Rocha, D.R.T.W. and Arbex, A.K. (2017) Pathophysiology of Graves' Ophthalmopathy: A Literature Review. Open Journal of Endocrine and Metabolic Diseases, 7, 77-87.

http://dx.doi.org/10.4236/ojemd.2017.71008

Received: November 11, 2016

Accepted: January 14, 2017

Published: January 17, 2017

Copyright $\odot 2017$ by authors and Scientific Research Publishing Inc. This work is licensed under the Creative Commons Attribution International License (CC BY 4.0).

http://creativecommons.org/licenses/by/4.0/

\begin{abstract}
Graves' ophthalmopathy (GO), an autoimmune condition associated with Graves' disease (GD), occurs at a prevalence of nearly $40 \%$ in patients diagnosed with GD. Ocular involvement is probably due to the presence of autoantibodies in the orbital tissues, regardless of the control state of thyroid hormones in individuals with GD, even during euthyroid or hypothyroid states, which are associated with thyroid hormone treatment. In addition to the immunological role present in the pathophysiology of GO, the genetic component, proteins and cytokines, minerals (e.g., selenium), and environmental factors (e.g., smoking) also contribute to its development and occurrence of clinical manifestations in varying degrees. Until now, the interaction of causal, intermediary, and triggering factors of GO is still unclear, so the purpose of this article is to review literature on the theme.
\end{abstract}

\section{Keywords}

Ophthalmopathy, Graves Disease, Pathophysiology

\section{Introduction}

Graves' ophthalmopathy (GO), an autoimmune condition associated with Graves' disease (GD), occurs at a prevalence of nearly $40 \%$ in patients diagnosed with GD [1] [2] [3] [4]. It is usually seen in people who have had GD for some time, and thus are also likely to be included in a more advanced age group than those who do not present with ocular changes. GO is more common in women, often associated with autoimmune disease, and has an incidence of 16.3 per 100,000 people/year. According to Tellez et al., the risk of developing GO is 6.4 times higher in the European population than that in the Asian population. Severe cases of GO represent $3 \%-5 \%$ of all cases and are more severe in men [1] 
[2] [3].

Ocular involvement is probably due to the presence of autoantibodies in the orbital tissues, regardless of the control state of thyroid hormones in individuals with GD. GO not only occurs during a hyperthyroid state, but also during euthyroid or even hypothyroid states, which are associated with thyroid hormone treatment [1] [4]. In addition to the still unclear immunological role present in the pathophysiology of GO (the main cells involved are the B lymphocytes, $\mathrm{T}$ lymphocytes, and orbital fibroblasts), the genetic component, proteins and cytokines, minerals (e.g., selenium), and environmental factors (e.g., smoking) also contribute to its development and occurrence of clinical manifestations in varying degrees [1] [2] [3] [4].

In view of the complex and little-known interaction of causal, intermediary, and triggering factors of GO, it is noticeable that the hypotheses are still many and that there are few certainties about the mechanism of pathophysiological changes in this condition. The purpose of this article is to review literature on the theme.

\section{Method}

This literature review was conducted through searches of the PubMed and Cochrane Library databases. The following keywords were used: ophthalmopathy, orbital, physiopathology, and Graves. Articles published in English from 2005 to 2016 were included.

\section{Genetics}

According to a population-based study with monozygotic Danish brothers, the concordance rate for the onset of GO was $35 \%$. It was also found that $30 \%-60 \%$ of affected individuals had a positive family history for this condition [1]. According to previous research that reported a higher prevalence of GO in the European population than in the Asian population, it can also be determined on a broader scale that there is an important genetic role, which contributes to the onset of this pathology. However, to date, it is still not possible to state with certainty which particular isolated or associated genes determine the occurrence and form the presentation of GO. This is because in different populations along the evolutionary process, there are infectious factors inherent to each geographical region, which may lead to polymorphic immune responses. The subsequent autoimmune attacks suffered by these individuals may be connected to different susceptible genes or groups of genes [1] [3].

According to Jacobson and Tomer, most of the genes that impact the development of GD are part of the body's immune system such as signaling receptors, molecules, and peptides [3]. The distribution of the polymorphism of the peroxisome proliferator Pro(12)Ala gene associated with the y receptor has been revised as a genetic predisposition to adipogenesis in GO. Such polymorphism was found to be equally present in GD patients with or without ocular involvement, and in patients with GO, its presence corresponded to a lower activity and severity 
of the disease [3]. According to Ban et al. (2016), in a study with the Japanese population, there was an association of GO with the single nucleotide polymorphism rs2358994 with the BCL2L15 gene [5]. On the other hand, in research with patients from the city of Malmo, Sweden, there were 10 single nucleotide polymorphisms in 4 genes: BTG2, CYR61, ZFP36, and SCD [6]. As GO is related to the immune system, several immunomodulatory genes have been reported as participants in this process: human leukocyte antigen (HLA) DR3, HLA-B8, cytotoxic T lymphocyte antigen (CTLA-4), interleukin-1, IL-23 receptor, CD40, protein tyrosine phosphatase, PTPN22, T cell receptor beta, tumor necrosis factor beta (TNF beta), and several genes associated with heavy chain immunoglobulins [2] [3].

Also with regard to the severity of $\mathrm{GO}$, it was observed that it occurs more significantly in individuals with HLA-B8, DR3, and DQA 0501 haplotype, whereas HLA-DR $B 1^{\star} 07$ seems to offer protection, reducing the susceptibility to the development of GO [1] [3]. Akaishi et al. observed in a study with 81 Brazilian patients with GO and 161 normal controls that the extraocular muscle involvement was more frequent with allele 16 of the HLA-DRB1 and lower with allele 03 of the HLA-DRB1 [1]. An association between higher serum rate of CTLA-4 AG and a polymorphism at codon 17 of this protein was also related to the degree of severity, but this finding did not appear in cohort studies with larger numbers of patients with the same genetic characteristics [3]. The polymorphisms found more often were the $I L-1$ alfa889C/T (odds ratio of 5.7 for TT genotype) and IL-1 receptor antagonist (IL-1RA) with Mspa-1 11100C/T (odds ratio of 6.7 for CC genotype) in patients with GO when compared to controls [3]. The genes encoding the TSHR (thyrotropin receptor) are being investigated in the same way. These genes are also related to the severity of GO and PPAR gamma, which is related to the adipogenesis mechanism that occurs in the orbit of affected individuals [3]. Regarding IL-23, two polymorphisms were detected (rs10889677 and rs2201841), which occur at a rate 1.8 higher than in those unaffected by GO [3]. It also seems to be a gene upregulation relationship involved in the innate, cell-mediated, and inflammatory immune responses in the GO orbital adipogenesis (TIMD4, DEFA1B, DEFA1, and DEFA3) [7].

\section{Immunopathogenesis}

1) Lymphocytes

$\mathrm{B}$ and $\mathrm{T}$ lymphocytes are essential in immune processes. B lymphocytes (mostly subtypes B1 and B2), proliferate, migrate, and produce antibodies when foreign antigens are found. This sequence of events basically happens in two ways: B lymphocytes are activated directly by their own antigens or bind, through the CD40 protein found on their surface, with the CD40 binder present, in turn, on the surface of T lymphocytes. This interaction allows $\mathrm{T}$ lymphocytes to produce cytokines and restimulate B lymphocytes to produce specific immunoglobulins [4] [8].

The way in which $\mathrm{T}$ lymphocytes recognize antigens is through their surface 
receptors. They recognize specific peptides that are bound to major histocompatibility complex proteins (MHC) and on the surface of antigen presenting cells, among them macrophages, B cells, and dendritic cells [2] [8]. For the T lymphocytes to be activated, there are two types of signals. The first signal is when the antigen-MHC complex targets the T lymphocyte receptors. The second happens when the $\mathrm{B} 7$ molecule from the surface of an antigen presenting cell, in this case probably a B lymphocyte, binds to the $\mathrm{CD} 28$ of the T lymphocyte surface [8].

The activation of $\mathrm{T}$ lymphocytes leads to the proliferation and differentiation of a particular type of effector cell. If one of the signals responsible for the activation of $\mathrm{T}$ cells is absent, these cells are not able to be activated. This phenomenon, called peripheral anergy, is one of the main tolerance mechanisms in the body, preventing the development of autoimmunity. However, these defense mechanisms often fail, making room for the onset of autoimmunity [8].

T lymphocytes of the Th17 subtype are related to several autoimmune diseases such as multiple sclerosis, arthritis, and psoriasis. In GO, these cells migrate to the orbit and produce cytokines such as IL-23R, whose variants are strongly associated with the onset of this condition; however, the mechanisms of its origin have not yet been determined. Th17 lymphocytes will also stimulate B lymphocytes in the orbit to produce autoantibodies against autoantigens such as TSH-R and IGF-1R, which originated, in turn, from the orbital fibroblasts [8]. A larger population of FoxP3-positive and -negative CD4(+) CD25(+) T cells was also observed in patients with the development of GO [9].

2) Fibroblasts

TSH-R normally exists on the surface of orbital fibroblasts, but immunoreactivity is only present in GO, whereas IGF-1R appears with higher serum levels in affected individuals [2]. The fibroblasts stimulated by autoantibodies against IGF-1R will produce cytokines such as IL-16, RANTES, and CXCL10 that will be responsible for attracting more $\mathrm{T}$ lymphocytes, which, in turn, will originate more cytokines, such as IFN $\gamma, \mathrm{TNF} \alpha$, PGD2, and 15d-PGJ2 [8]. In view of this process, the fibroblasts are stimulated to synthesize extracellular matrix molecules such as hyaluronic acid and other fibroblasts, and to induce the accumulation of adipose tissue in the orbit, leading to the changes recognized in GO [1]. Orbital fibroblasts comprise a heterogeneous population of cells that can be differentiated by their surface markers. Several studies have used Thy-1, a fibroblast subtype that differentiate intomyofibroblasts (Thy-1+) or adipocytes (Thy-1-). The origin of this variety of cells in connective tissues is still undefined, but it is known that they are fundamental for the remodeling seen in different parts of the body when exposed to particular factors, such as inflammation. In the orbit, for example, Thy- 1 fibroblasts differentiate into adipocytes, which explains the expansion of the orbital volume in GO [4] [8].

Fibrocytes are monocyte lineage cells that are present in the bone marrow, present antigens against lymphocytes and can enter the tissue injury sites. They differ from fibroblasts, monocytes, $\mathrm{T}$ and $\mathrm{B}$ lymphocytes, epithelial, endothelial, 
and dendritic cells, and can differentiate into mature cells as adipocytes, osteoblasts, and myofibroblasts. This differentiation depends partly on the signal received and coming from the tissue microenvironment in which they will have to start a specific remodeling [8]. Apparently, fibrocytes resemble fibroblasts. Once fibrocytes infiltrate into the tissue that has suffered injury and initiate a remodeling, they assume a fibroblast-like morphology, losing the CD34 expression on their surface, which suggests that this protein is a progenitor marker [2].

GD is an autoimmune process in which the thyroid becomes enlarged and hyperactive in its hormone production. This excess production involves an increased autoantibody activity against the TSHR. In addition, the IGF-1 receptor (IGF-1R) is over expressed in the orbital fibroblasts and in the T and B lymphocytes of the affected individuals. IGF-1R is a second-line potential pathogenic autoantigen for the contribution to the increase in the thyroid and lymphocyte migration to the affected tissues, such as the pretibial connective tissue and orbit tissue [2] [8].

In a study conducted by Lehmann et al. [8], it is suggested that the displacement of $\mathrm{T}$ lymphocytes to the orbit may be mediated by an immune response of fibroblasts to the immunoglobulin G of GD (IgG-GD). These fibroblasts then express high levels of T lymphocyte chemokines, IL-16, and RANTES [8]. In addition to the chemokines, the fibroblasts found in the orbit of patients with GO synthesize large amounts of connective tissue growth factor (CTGF), hyaluronic acid in response to IgG-GD and IGF-1, which accumulates and causes local tissue edema [4] [10]. The connective tissue found in the orbit is highly prone to inflammation, which accounts for approximately $30 \%$ of patients with GD developing an important component of clinical manifestations that constitute GO, where the connective tissue is inflamed with infiltrates of $\mathrm{T}$ and $\mathrm{B}$ lymphocytes and mastocytes. GO-mediator chemokines and cytokines seem to activate local fibroblasts which present a unique phenotype [1] [4].

The fibroblasts of the orbit affected by GO consist of a heterogeneous population of cells and show a strong response to pro-inflammatory cytokines, such as those from the IL-1 family. When activated by IL-1B and CD154, these cells produce hyaluronic acid, resulting in the induction of hyaluronan synthase (HAS) 1,2, and 3, and uridyltransferase glucose dehydrogenase. This exaggerated induction of HAS isoforms will lead to hyaluronic acid accumulation in GO [4]. When activated, orbital fibroblasts also express IL-6, IL-8, and prostaglandinendoperoxide synthase 2 , the inflammatory cyclooxygenase. This induction results in a higher production of prostaglandin E2 (PGE2). This prostanoid seems to play an important role in the immune response that occurs in the orbit, favoring the predominance of T lymphocyte helper type 2 [4]. Cao and Smith demonstrated that there is a low production of IL-1 receptor antagonists (IL-1 RA) by the fibroblasts of GO. Low levels of IL-1 RA result in low opposition to the inflammatory initial signal resulting from the production of IL- $1 \alpha$ and IL-1 $\beta$. The immune response that occurs in the orbital fibroblasts seems to be much more abundant than that observed in other phenotypes of these cells [4]. The 
wide variety of existing types of fibroblasts cannot yet be well explained.

Regarding the types that can or cannotproduce Thy-1 (CD90), these cells can be categorized among those that have the potential to differentiate into adipocytes (Thy-1-) or myofibroblasts (Thy-1+) [4]. The fibroblasts designed to be adipocytes can differentiate spontaneously or in a more facilitated manner when they are exposed to prostacyclin along with components that enhance cAMP levels or with molecules that activate the PPAR $\gamma$ [4]. On the other hand, the Thy-1+ fibroblasts that will differentiate into myofibroblasts and express high levels of muscle actin do so when exposed to TGF- $\beta$. Simultaneous to these differentiations, there is an increase of the TSHR, but it is still not possible to determine which of the two types of fibroblasts, Thy-1- or Thy-1+, is related to this increase [4].

Douglas et al. observed that the pool of circulating fibrocytes in the patients with GD is much higher than in healthy subjects. As well as in other studies, it was also found that fibrocytes derived from cultures of peripheral blood mononuclear cells are rare in patients with any chronic disease known [11]. On the other hand, patients with GD, regardless of duration, thyroid hormone levels, or pathology treatment time, have numerous fibrocytes originated by peripheral blood mononuclear cells that exhibit the phenotype CXCL-12/CXCR4 [9] [12]. In contrast to the previous statement, not all individuals with GD have an increased level of circulating fibrocytes and fibroblasts compared to the controls, as well as the levels of IGF-1R [2] [13].

Douglas et al. started characterizing phenotypes of fibrocytes involved in GO in their studies. CD $34^{+}$Col1 $1^{+}$IGF- $1 \mathrm{R}^{+}$cells are abundant in the orbit tissues of those affected by GO and are absent in controls. These cells are also CD31-, which indicates they are not related to endothelial cells [13]. High levels of TSHR were also detected on the surface of fibrocytes. These levels were similar to those found in the epithelial cells of the thyroid, where hormone production is mediated. More importantly, they were also functional [9]. When related to a bovine thyroid stimulating hormone (bTSH) or M22, which constitutes a monoclonal antibody against TSHR, the production of inflammatory cytokines such as TNF- $\alpha$ and IL-6 is significantly upregulated [13]. In patients with GO, when they had their fibroblasts submitted to cytometric analysis, the cell subtype $\mathrm{CD} 34+\mathrm{Col1}+$ was found. On the other hand, CD34+ lymphocytes were significantly absent of the sample of fibroblasts from control subjects, with the vast majority of these cells also showing the CD90+ phenotype (Thy-1+) on their surface [13].

3) TSHR

There is agreement among several studies presenting different methodologies that TSHR mRNA in the orbital adipose tissue is present in both healthy individuals and those with GO [3] [4]. It is known that the TSHR expression is larger in affected people, with a positive correlation between their serum levels and clinical scores of disease presentation. Despite the knowledge of this existence, it is not known how the anti-TSHR antibodies (Trab) act in signaling the TSHR in 
the orbital tissues and dermal fibroblasts. One suggestion is that there are different subtypes of Trab, still unable to be detected by the methods of existing studies and that are responsible for the development of different affections of orbital and dermal tissues [2] [3] [4]. Eckstein et al. concluded, for example, that serum Trab values were still detectable in patients with GO who responded unsatisfactorily to anti-inflammatory therapy and were undetectable in good responders to treatment [3].

\section{Biochemical Factors}

1) Thyroxine and Triiodothyronine

As for serum thyroxine (T4) and triiodothyronine (T3) values in the role of development or worsening of GO, there is much controversy when analyzing several recent studies, especially when there is comparison between research involving the Asian and non-Asian populations, a fact which refers once again to the importance of genetic factors in the etiopathogenesis and clinical course of the disease [3].

2) Tobacco

In view of all non-genetic factors exposed as contributing to the onset and worsening of GO, smoking is the most consistent and least controversial among studies [1] [3] [14]. In a study with newly diagnosed patients with GD and treated with radioiodine or antithyroid drugs, smoking was the biggest risk factor for the development of GO, with an odds ratio of 5.2 (95\% CI: 2.4 - 11.5). Subsequently, a new, more detailed analysis was applied to define the onset criteria of GO. In this second phase of the study the cigarette proved to be even more related to GO, with an odds ratio of 9.8 (95\% CI: 2.9 - 34.9) [3]. An important detail is that the choice of treatment for GD in smokers did not influence the risk of onset of GO, although in previous research this risk was higher in patients with GD who smoked and were subjected to treatment with radioiodine. These patients demonstrated a 50\% risk of progressing to GO in a 3-year period [3]. The possibility of development and worsening of the disease is higher in smokers when compared to controls, regardless of the type of treatment used for normalizing hyperthyroidism: iodine therapy, use of antithyroid drugs and/or corticosteroids [3]. The risk is also directly proportional to the number of cigarettes smoked a day [3].

In Europe, an investigation was conducted by means of questionnaires directed to children under 10 years of age about the relationship between GO and secondhand smoke, taking into account that the prevalence of GO in the population of this age group in these different countries was similar. The ratio was found to be $52 \%$ among children with ocular involvement when the prevalence of smoking in the countries was greater than $25 \%$, whereas it was $19 \%$ in countries with a lower prevalence of this habit [3].

Currently, there are no prospective studies to confirm the relationship of smoking with the etiopathogenesis of GO, only its contribution to the worsening of symptoms and worse clinical response to treatment. However, evidence from 
several retrospective research studies have demonstrated an association between smoking and onset of GO [3].

1) Thyroglobulin

The hypothesis of the role of thyroglobulin as a GO mediator began with the studies by Konishi et al. [12] and McDougall and Kriss [13], which suggested that the thyroid thyroglobulin would migrate to the orbits by lymphatic pathways, where it would be recognized by anti-thyroglobulin antibodies, causing the orbital inflammation. Fifteen years later, Martino et al. identified intact thyroglobulin in the retro-orbital tissues of three patients with GO, however the thyroglobulin anti-thyroglobulin complexes were not observed, so these authors remained with the hypothesis of cell-mediated autoimmunity as the responsible for the pathophysiology of GO [15].

2) Proteins

Matheis et al. (2015) found 10 proteins present in upregulation in the orbital tissue of untreated patients with GO: beta IV spectrin (6.2-fold), GTP binding G protein 2 (5.6-fold), POTE ankyrin domain family member F (5.4-fold), xylulokinase (4.1-fold), kinesin family member 1A and lipocalin 1 (both 3.6-fold), semicarbazide-sensitive metalloproteinase amine oxidase 3 and polymerase I transcript release factor (both 3.4-fold), cell-cycle protein elongin A binding protein 1 (3.3-fold), annexin A2 andcavin (both 3-fold), protein pointing to cell proliferation histone $\mathrm{H} 4$ (2.8-fold), and ADAM metallopeptidase with thrombospondin type 1 motif 14 (2.7-fold) [16].

Mechanical factors

Proptosis occurs due to a displacement of the eyeball to the orbital anterior region, due to the increase in volume of the orbital fat and extraocular muscles present in GO inflammatory process. This mechanism happens as a compensatory process so that normal intraorbital pressure is reestablished [3]. Patients with GO seem to have a wider angle of the lateral orbital wall as compared to controls, a fact that also occurs in the non-inflammatory proptosis, called nonsyndromic exophthalmia [3]. These anatomical variations of orbital contours, as well as its web variations of veins and lymphatic vessels, in part seem to explain why sometimes there is an asymmetrical ocular involvement in the same person [3]. In addition, individuals who have higher degrees of myopia determine more pronounced proptosis when compared to those with GO without myopia [3].

Radioiodine therapy

Several retrospective cohort studies and randomized trials demonstrate the radioiodine therapy as a risk factor for the development and aggravation of GO, with a rate of $15 \%-39 \%$ [3]. These rates would be $38.7 \%$ when radioiodine therapy is compared to the use of antithyroid drugs and $16 \%$ when compared to surgery [3]. However, such studies are greatly inconsistent, since different parameters are used to define the development of GO and lack well defined criteria for disease activity assessment [3].

Selenium

Selenium participates in various biological processes in the human body. The 
selenium-dependent enzymes are generally antioxidative and anti-inflammatory [2] [17] [18]. Glutathione peroxidase can reduce hydrogen peroxides and phospholipid peroxides, thereby reducing free radicals and reactive oxygen species. The low circulating concentration of hydroperoxides decreases the production of inflammatory prostaglandins and leukotrienes. This whole sequence of events contributes to reducing the thyroid autoimmune inflammatory activity response [18]. Its effects are mostly as an antioxidant, by means of the selenoproteins $\operatorname{Trx}$ reductase and GPx [18]. Trx is a stress- and iodine-induced protein, which has strong redox activities and appears increased in GD, being expressed in thyroid follicular cells. Selenium, by means of Trx reductase, acts by inhibiting the formation of Trx, reducing its inflammatory action [18].

In the hypermetabolic acute state of the GD, there is an increased update of oxygen and intracellular ATP, leading to dysfunction of the mitochondria and the appearance of free radicals that lead to cell injury. When GPx is activated, the selenium activates a "second line" of antioxidant defense, in addition to the first line represented by superoxide dismutase and catalase enzymes, capable of neutralizing the biological effects of free radicals [18]. These reactive oxygen species, as well as hydrogen peroxide, can stimulate the NF-kB pathway, a major pathway responsible for the inflammatory immune response and associated with an increase of TNF-a and IL-6. Selenium inhibits NF-kB to bind to its gene promoters and consequently reduces the production of cytokines, reducing inflammation [18]. Experimental studies on hyperthyroidism show that, in this state, there is an increase in Trx and Gpx systems, stimulated by the phosphatidylinositol calcium cascade, as well as an increase of superoxide dismutase and glutathione in erythrocytes. Thus, when this process gets longer due to the hyperthyroidism time, it can suffer failure, requiring a selenium dietary supplementation [19].

Ashok et al. conducted a double-blind randomized study with 60 patients with autoimmune thyroid diseases, all with anti-TPO greater than $150 \mathrm{IU} / \mathrm{mL}$. For 2 months, 30 patients received a daily dose of $200 \mu \mathrm{g}$ of selenium and the other 30 received placebo. The study excluded pregnant women and patients who already were receiving clinical treatment, such as use of antithyroid drugs or any other drug that could influence the autoimmune response. Of the patients treated with selenium, 6 became hypothyroid, another 15 had subclinical hypothyroidism, 6 normalized their levels of thyroid hormones, and 3 had subclinical hyperthyroidism. In the group treated with selenium, the concentration of anti-TPO decreased significantly by $49.5 \%(\mathrm{P}<0.013)$ [19]. In a prospective clinical study with controlled use of selenium and placebo in Bavaria (southeastern area of Germany), Gärtner et al., in 2002, reported a 36\% reduction in the anti-TPO titers of the selenium group. This decrease was even greater among patients with baseline levels of anti-TPO above $1200 \mathrm{IU} / \mathrm{mL}$ [19].

\section{FOXOs}

Zhang et al. (2016) stated that the Forkhead transcription factors (FOXOs) play an important role in regulating adipogenesis of fibroblasts and pre-adipo- 
cytes of the orbit of patients with GO, because when treating these cells with the FOXO inhibitor AS1842856 or with its enhancer trifluoperazine hydrochloride (TFP), found that the increase in FOXOs was related to a lower orbital adipogenesis, and these were possible alternatives for the development of new immunosuppressive therapies [20].

Although there are many studies in literature on the various mechanisms that may be present in the development of GO, there is still no consensus on the actual role of each one within the complex pathophysiological cascade of this disease.

\section{References}

[1] Maheshwari, R. and Weis, E. (2012) Thyroid Associated Orbitopathy. Indian Journal of Ophthalmology, 60, 87-93. https://doi.org/10.4103/0301-4738.94048

[2] Gupta, S. and Douglas, R. (2011) The Pathophysiology of Thyroid Eye Disease (TED): Implications for Immunotherapy. Current Opinion in Ophthalmology, 22, 385-390. https://doi.org/10.1097/ICU.0b013e3283499446

[3] Stan, M.N. and Bahn, R.S. (2010) Risk Factors for Development or Deterioration of Graves' Ophthalmophaty. Thyroid, 20, 777-783. https://doi.org/10.1089/thy.2010.1634

[4] Naik, V.M., Naik, M.N., Goldberg, R.A., Smith, T.J. and Douglas, R.S. (2010) Immunopathogenesis of Thyroid Eye Disease: Emerging Paradigms. Survey of Ophthalmology, 55, 215-226. https://doi.org/10.1016/j.survophthal.2009.06.009

[5] Ban, Y., Tozaki, T. and Nakano, Y. (2016) Association Studies of the GPR103 and BCL2L15 Genes in Autoimmune Thyroid Disease in the Japanese Population. Frontiers in Endocrinology (Lausanne), 7, 92. https://doi.org/10.3389/fendo.2016.00092

[6] Planck, T., Shahida, B., Sjögren, M., Groop, L., Hallengren, B. and Lantz, M. (2014) Association of BTG2, CYR61, ZFP36, and SCD Gene Polymorphisms with Graves' Disease and Ophthalmopathy. Thyroid, 24, 1156-1161.

https://doi.org/10.1089/thy.2013.0654

[7] Khong, J.J., Wang, L.Y., Smyth, G.K., McNab, A.A., Hardy, T.G. and Selva, D. (2015) Differential Gene Expression Profiling of Orbital Adipose Tissue in Thyroid Orbitopathy. Investigative Ophthalmology \& Visual Science, 56, 6438-6447. https://doi.org/10.1167/iovs.15-17185

[8] Lehmann, G.M., Feldon, S.E., Smith, T.J. and Phipps, R.P. (2008) Immune Mechanisms in Thyroid Eye Disease. Thyroid, 18, 959-965.

https://doi.org/10.1089/thy.2007.0407

[9] Matsuzawa, K., Izawa, S., Okura, T., Fujii, S., Matsumoto, K., Shoji, K., et al. (2016) Implications of FoxP3-Positive and -Negative CD4 ${ }^{+} \mathrm{CD} 25^{+} \mathrm{T}$ Cells in Graves' Ophthalmopathy. Endocrine Journal, 63, 755-764. https://doi.org/10.1507/endocrj.EJ16-0108

[10] Huang, Y.M., Chang, P.C., Wu, S.B., Kau, H.C., Tsai, C.C., Liu, C.J., et al. (2016) Expression and Clinical Significance of Connective Tissue Growth Factor (CTGF) in Graves' Ophthalmopathy. British Journal of Ophthalmology, Article ID: bjophthalmol-2016-308713.

http://bjo.bmj.com/content/early/2016/08/19/bjophthalmol-2016-308713.long

[11] Douglas, R.S., Afifiyan, N.F., Hwang, C.J., Chong, K., Haider, U., Richards, P., et al. (2010) Increased Generation of Fibrocytes in Thyroid-associated Ophthalmopathy. The Journal of Clinical Endocrinology \& Metabolism, 95, 430-438. https://doi.org/10.1210/jc.2009-1614 
[12] Fernando, R., Atkins, S.J. and Smith, T.J. (2016) Intersection of Chemokine and Thyrotropin Receptor Pathways in Human Fibrocytes: Emergence of CXCL-12/ CXCR4 Crosstalk Potentially Relevant to Thyroid-Associated Ophthalmopathy. Endocrinology, 157, Article ID: en20161382. http://press.endocrine.org/doi/10.1210/en.2016-1382

[13] Smith, T.J. (2010) Potential Role for Bone Marrow-Derived Fibrocytes in the Orbital Fibroblast Heterogeneity Associated with Thyroid-Associated Ophthalmopathy. Clinical \& Experimental Immunology, 162, 24-31. https://doi.org/10.1111/j.1365-2249.2010.04219.x

[14] Wiersinga, W.M. (2012) Graves' Orbitopathy: Management of Difficult Cases. Indian Journal of Endocrinology and Metabolism, 16, S150-S152.

[15] Shanmuganathan, T., Girgis, C., Lahooti, H., Champion, B. and Wall, J.R. (2015) Does Autoimmunity against Thyroglobulin Play a Role in the Pathogenesis of Graves' Ophthalmopathy: A Review. Clinical Ophthalmology, 9, 2271-2276.

[16] Matheis, N., Lantz, M., Grus, F.H., Ponto, K.A., Wolters, D. and Brorson, H. (2015) Proteomics of Orbital Tissue in Thyroid-Associated Orbitopathy. The Journal of Clinical Endocrinology \& Metabolism, 100, E1523-E1530. https://doi.org/10.1210/jc.2015-2976

[17] Weeks, B.S. and Hanna, M.S. (2012) Dietary Selenium and Selenoprotein Function. Medical Science Monitor, 18, RA127-RA132. https://doi.org/10.12659/MSM.883258

[18] Duntas, L.H. (2012) The Evolving Role of Selenium in the Treatment of Graves' Disease and Ophthalmopathy. Journal of Thyroid Research, 2012, Article ID: 736161. https://doi.org/10.1155/2012/736161

[19] Bhuyan, A.K., Sarma, D. and Saikia, U.K. (2012) Selenium and the Thyroid: A Close-Knit Connection. Indian Journal of Endocrinology and Metabolism, 16, S354-S355.

[20] Zhang, L., Ji, Q.H., Ruge, F., Lane, C., Morris, D., Tee, A.R., et al. (2016) Reversal of Pathological Features of Graves' Orbitopathy by Activation of Forkhead Transcription Factors, FOXOs. The Journal of Clinical Endocrinology \& Metabolism, 101, 114-122. https://doi.org/10.1210/jc.2015-2932

Scientific Research Publishing

Submit or recommend next manuscript to SCIRP and we will provide best service for you:

Accepting pre-submission inquiries through Email, Facebook, LinkedIn, Twitter, etc. A wide selection of journals (inclusive of 9 subjects, more than 200 journals)

Providing 24-hour high-quality service

User-friendly online submission system

Fair and swift peer-review system

Efficient typesetting and proofreading procedure

Display of the result of downloads and visits, as well as the number of cited articles

Maximum dissemination of your research work

Submit your manuscript at: http://papersubmission.scirp.org/

Or contact ojemd@scirp.org 